\title{
Light-dependent ammonium ion toxicity of rice leaves in response to phosphinothricin treatment
}

\author{
Y.-C. TSAI and C.H. KAO \\ Department of Agronomy, National Taiwan University, \\ Taipei, 106, Taiwan, Republic of China
}

\begin{abstract}
Ammonium ion accumulation in detached rice leaves treated with phosphinothricin (PPT), an inhibitior of glutamine synthetase (GS), was investigated in the light and darkness. PPT treatment increased $\mathrm{NH}_{4}^{+}$content and induced toxicity in rice leaves in the light but not in darkness, suggesting the importance of light in PPT-induced $\mathrm{NH}_{4}{ }^{+}$toxicity in detached rice leaves. PPT treatment in the light resulted in a decrease of activities of the cytosolic form of GS and the chloroplastic form of GS. The photosynthetic electron transport inhibitor 3-(3,4-dichlorophenyl)-1,1-dimethylurea reduced $\mathrm{NH}_{4}{ }^{+}$accumulation induced by PPT in the light. In darkness, PPT-induced $\mathrm{NH}_{4}^{+}$accumulation and toxicity were observed in the presence of glucose or sucrose.
\end{abstract}

Additional key words: glutamine synthetase, Oryza sativa.

\section{Introduction}

Ammonium ion is a central intermediate of nitrogen metabolism in plants. Glutamine synthetase (GS, EC 6.3.1.2) plays a crucial role in the assimilation of ammonium. In most plants the GS/GOGAT (glutamate synthase) pathway is the only efficient way to detoxify the $\mathrm{NH}_{4}^{+}$released by nitrate reduction, amino acid degradation or photorespiration (Miflin and Lea 1976). Thus, plants are very susceptible to inhibitors of GS (Lydon and Duke 1999). Among GS inhibitors, phosphinothricin [PPT, 2-amino-4-(methyl phosphinyl)butanoic acid, also known as glufosinate] has received special attention (Miflin and Lea 1976). PPT is known to bind to the active site of GS in competition with glutamate (Lea and Ridley 1990). After the PPT is applied, a rapid accumulation of $\mathrm{NH}_{4}^{+}$is observed (Tachibana et al. 1986, Wild et al. 1987). A high content of $\mathrm{NH}_{4}{ }^{+}$is known to have a toxic effect on plant cells (Givan 1979). It has been shown that PPT-induced $\mathrm{NH}_{4}^{+}$ accumulation is light-dependent in tomato and Sinapis alba (Perez-Garcia et al. 1998, Wild et al. 1987). It is not known whether PPT-induced $\mathrm{NH}_{4}{ }^{+}$accumulation is also light-dependent in rice leaves. The aim of this study was to examine the effect of PPT on ammonium contents and toxicity in detached rice leaves in the light and darkness.

\section{Materials and methods}

Rice (Oryza sativa L. cy. Taichung Native 1) seedlings were planted on a stainless net floating on half-strength Johnsons's modified nutrient solution (Johnson et al. $1957)$ in a $500-\mathrm{cm}^{3}$ beaker. The nutrient solution $(\mathrm{pH} \mathrm{4.8)}$ was replaced every three days. Rice plants were grown for $12 \mathrm{~d}$ in a greenhouse, under natural light and the day/night temperature of $30 / 25{ }^{\circ} \mathrm{C}$ (for detail see Lin et al. 1999). The apical $3 \mathrm{~cm}$ of the third leaf of 12-d-old seedlings was used for the experiment. A group of 10 segments was floated in a Petri dish containing $10 \mathrm{~cm}^{3}$ of test solution. Incubation was carried out at $27^{\circ} \mathrm{C}$ in the light $\left(40 \mu \mathrm{mol} \mathrm{m} \mathrm{m}^{-2}\right)$ or in the dark.

Received 12 July 2001, accepted 5 December 2001.

Abbreviations: DCMU - 3-(3,4-dichlorophenyl)-1,1-dimethylurea; f.m. - fresh mass; GOGAT - glutamate synthase; GS - glutamine synthetase; GS1 - cytosolic form of GS; GS2 - chloroplastic form of GS; PPT - phosphinothricin.

Acknowledgements: This work was supported by the National Science Council of the Republic of China, grant NSC 89-2313-B-002-222.

Fax: (+886) 23620879, e-mail: kaoch@ccms.ntu.edu.tw 
The toxicity of detached rice leaves was followed by measuring the decrease of chlorophyll and protein contents. Chlorophyll was determined according to Wintermans and De Mots (1965) after extraction in $96 \%$ $(\mathrm{v} / \mathrm{v})$ ethanol. For protein determination, leaf segments were homogenized in $50 \mathrm{mM}$ sodium phosphate buffer (pH 6.8). The extracts were centrifuged at $17600 \mathrm{~g}$ for $20 \mathrm{~min}$, and the supernatants were used for determination of protein by the method of Bradford (1976). For $\mathrm{NH}_{4}{ }^{+}$ determination, leaf segments were homogenized in $0.3 \mathrm{mM}$ sulphuric acid $(\mathrm{pH} 3.5)$. The homogenate was centrifuged for $10 \mathrm{~min}$ at $39000 \mathrm{~g}$ and the supernatant was used for determination of $\mathrm{NH}_{4}{ }^{+}$, based on phenolhypochlorite reaction, by the method described previously (Lin and Kao 1996). For determination of glutamine, leaf samples were extracted with $2 \%$ sulfosalicylic acid and the homogenate was centrifuged at $15000 \mathrm{~g}$ for $20 \mathrm{~min}$. The supernatant was used directly for glutamine analysis by an amino acid analyzer (Beckman 6300, Palo Alto, CA, USA).

\section{Results and discussion}

The amount of $\mathrm{NH}_{4}{ }^{+}$increased significantly with increasing concentrations of PPT from 25 to $100 \mu \mathrm{M}$ under light. After $3 \mathrm{~d}$ of incubation under light, treatment

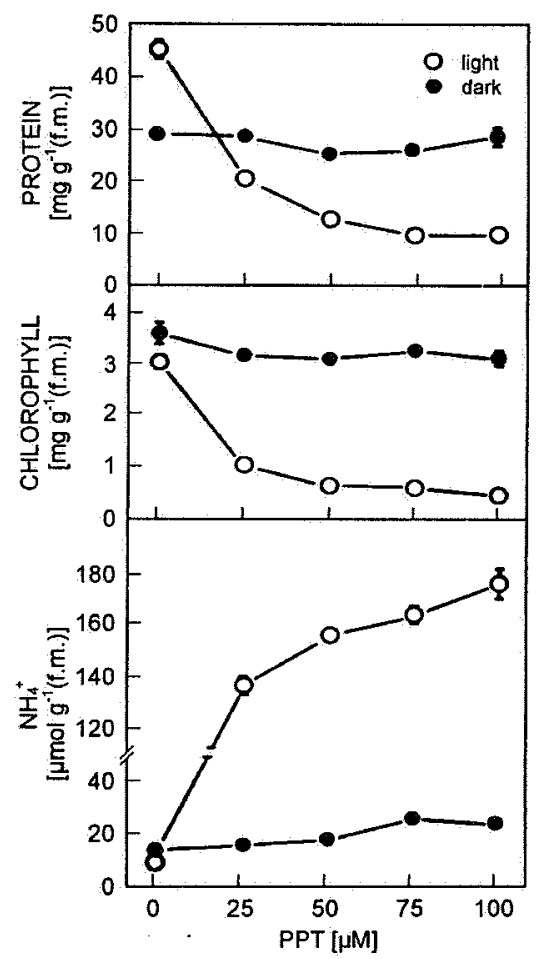

Fig. 1. Effects of PPT on protein, chlorophyll, and ammonium ion contents in detached rice leaves in the light and darkness. All measurements were made 3 days after treatment. Vertical bars represent standard errors $(n=4)$. Only those standard errors larger than the symbols are shown.
For extraction of GS, leaf segments were homogenized with $10 \mathrm{mM}$ Tris- $\mathrm{HCl}$ buffer $(\mathrm{pH} \mathrm{7.6,}$ containing $1 \mathrm{mM} \mathrm{MgCl}, 1 \mathrm{mM}$ EDTA and $10 \mathrm{mM}$ 2-mercaptoethanol) in a chilled pestle and mortar. The homogenate was centrifuged at $15000 \mathrm{~g}$ for $30 \mathrm{~min}$ and the resulting supernatant was used for determination of GS activity. The whole extraction procedure was carried out at $4{ }^{\circ} \mathrm{C}$. GS was assayed by the method of Oaks et al. (1980). One unit (U) of GS activity is defined as $1 \mu \mathrm{mol}$ L-glutamate-r-monohydroxamate formed per min. In order to separate the activities of GS1 and GS2 isoforms in leaf extract, activities were also measured in the same conditions in the presence of $1 \mathrm{mM}$ glucosamine-6phosphate, a specific inhibitor of GS2 (Hirel and Gadel 1980).

All experiments were repeated three times; within each experiment, treatments were replicated 4 times. Similar results and identical trends were obtained in add experiments. The data reported here are from a single experiment.

with $25 \mu \mathrm{M}$ PPT increased $\mathrm{NH}_{4}{ }^{+}$content about 14 -fold. However, increasing concentration of PPT from 25 to

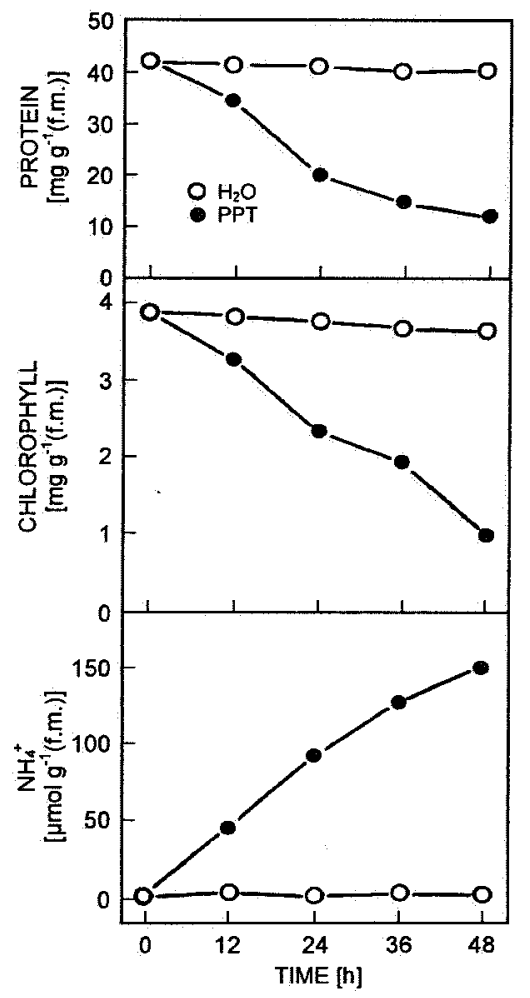

Fig. 2. Time courses of PPT effect on protein, chlorophyll, and ammonium ion contents in detached rice leaves in the light. Detached rice leaves were incubated in water or $50 \mu \mathrm{M}$ PPT. Vertical bars represent standard errors $(n=4)$. Only those standard errors larger than the symbols are shown. 
$100 \mu \mathrm{M}$ did not increase $\mathrm{NH}_{4}^{+}$content in detached rice leaves in darkness (Fig. 1). These results indicate that PPT-induced ammonium accumulation in detached rice leaves is light-dependent, which is consistent with the results reported by Perez-Garcia et al. (1998) and Wild et al. (1987).

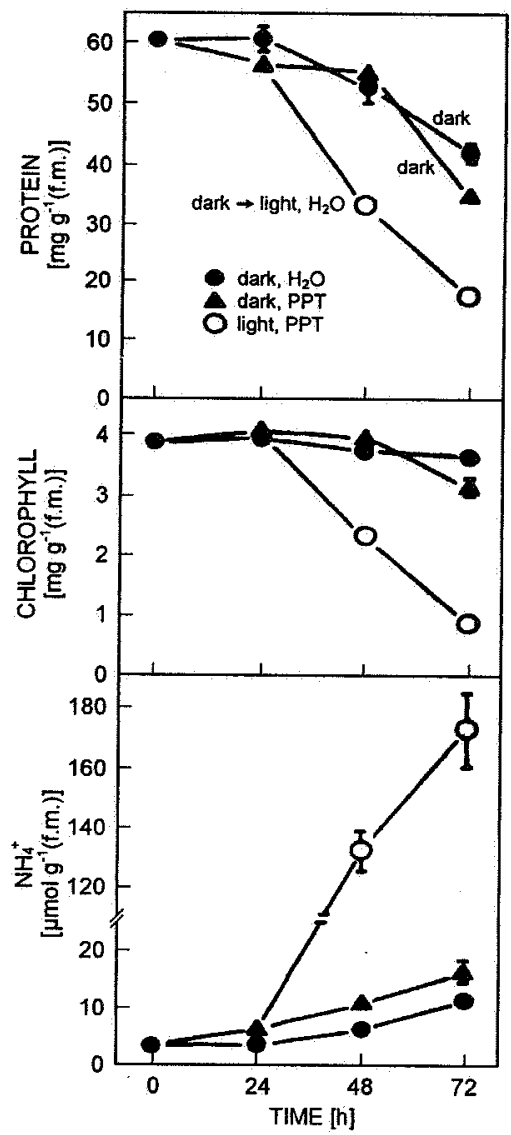

Fig. 3. Time courses for protein, chlorophyll, and ammonium ion contents in detached rice leaves treated with PPT in the light or darkness. The concentration of PPT was $50 \mu \mathrm{M}$. Vertical bars represent standard errors $(n=4)$. Only those standard errors larger than the symbols are shown.

A high content of $\mathrm{NH}_{4}{ }^{+}$is known to have a toxic effect on plant cells (Givan 1979). Thus PPT treatment is expected to cause toxicity in detached rice leaves, judged by the decrease of chlorophyll and protein, in the light but not in darkness. PPT treatment indeed resulted in a decrease of chlorophyll and protein contents in detached rice leaves in the light but not in darkness (Fig. 2).

Increase in $\mathrm{NH}_{4}^{+}$content, as a consequence of PPT treatment, was evident $12 \mathrm{~h}$ after the start of incubation in the light. Increased duration of treatment with PPT increased the content of $\mathrm{NH}_{4}{ }^{+}$. In untreated leaves, $\mathrm{NH}_{4}{ }^{+}$ content remained unchanged during the $48 \mathrm{~h}$ of incubation in the light. The toxicity caused by the treatment of PPT was evident at $12 \mathrm{~h}$ after treatment in the light. PPT-treated leaves, kept in the dark, showed a small increase in their $\mathrm{NH}_{4}^{+}$content and slight toxicity
(Fig. 3). When PPT and dark treated leaves were reilluminated, $\mathrm{NH}_{4}{ }^{+}$content was increased and toxicity became more severe (Fig. 3). These results clearly demonstrate the importance of light in $\mathrm{NH}_{4}^{+}$ accumulation and toxicity induced by PPT. DCMU in the light reduced the accumulation of $\mathrm{NH}_{4}{ }^{+}$and the toxicity induced by PPT (Fig. 4). For detached rice leaves incubated in the presence of glucose or sucrose under dark condition a greater PPT-induced accumulation of $\mathrm{NH}_{4}{ }^{+}$was observed. It seems that $\mathrm{NH}_{4}{ }^{+}$accumulation and toxicity induced by PPT in detached rice leaves in the light is modulated by photosynthetic activity. Since DCMU only partially reduced PPT-induced $\mathrm{NH}_{4}^{+}$ accumulation and toxicity in the light, and neither glucose nor sucrose increased $\mathrm{NH}_{4}{ }^{+}$and toxicity in detached rice leaves in darkness to the same extent as PPT in the light, one may conclude that light is required for one or more processes other than photosynthesis for maximum $\mathrm{NH}_{4}{ }^{+}$ accumulation and toxicity.

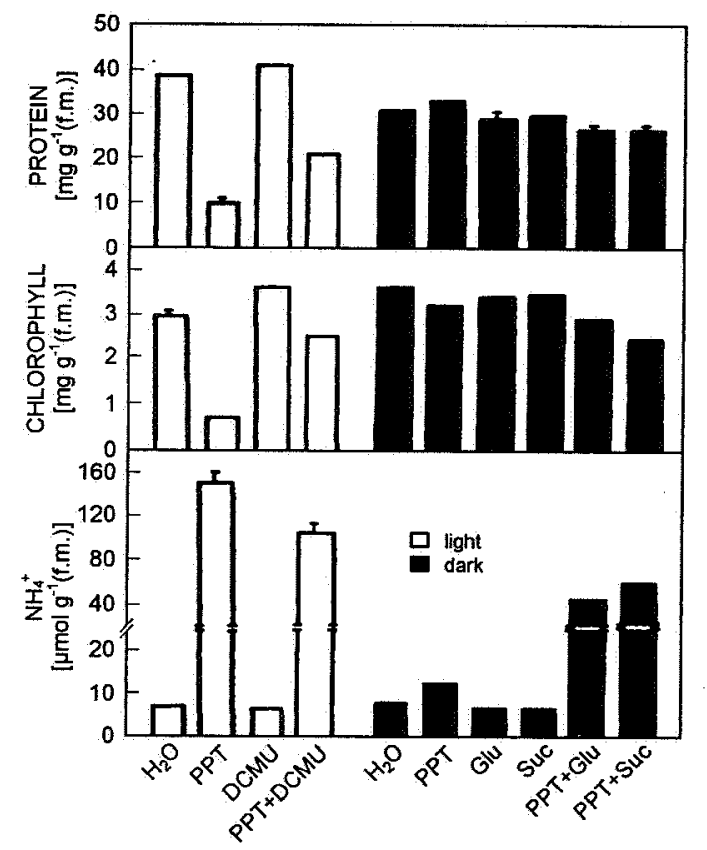

Fig. 4. Effects of DCMU on PPT-induced toxicity and ammonium ion accumulation in the light and of glucose and sucrose on protein, chlorophyll, and ammonium ion contents in detached rice leaves treated with PPT in darkness. The concentration of PPT, DCMU, glucose and sucrose were $50 \mu \mathrm{M}, 0.1 \mathrm{mM}, 100 \mathrm{mM}$, and $100 \mathrm{mM}$, respectively. All measurements were made 3 days after treatment. Vertical bars represent standard errors $(n=4)$.

In green tissues of angiosperms, the occurrence of the GS isoenzymes, GS1 and GS2, has been demonstrated (Lancien et al. 2000). GS1 catalyzes glutamine biosynthesis in the cytosol whereas GS2 is confined to the chloroplast (Lancien et al. 2000). Thus, it is of great interest to know the effect of PPT on total GS, GSI and GS2 activities in detached rice leaves in the light. As 
expected PPT decreased total GS activity in detached rice leaves in the light (Fig. 5). It was found that GS1 appears to be the predominant isoform in detached rice leaves of the test cultivar (Fig. 5). Lutts et al. (1999) also observed that GS1 was the predominant isoform in leaves of rice. It was also found that PPT was effective in decreasing both GS1 and GS2 activity (Fig. 5). Our results are inconsistent with those of Perez-Garcia et al. (1998), who

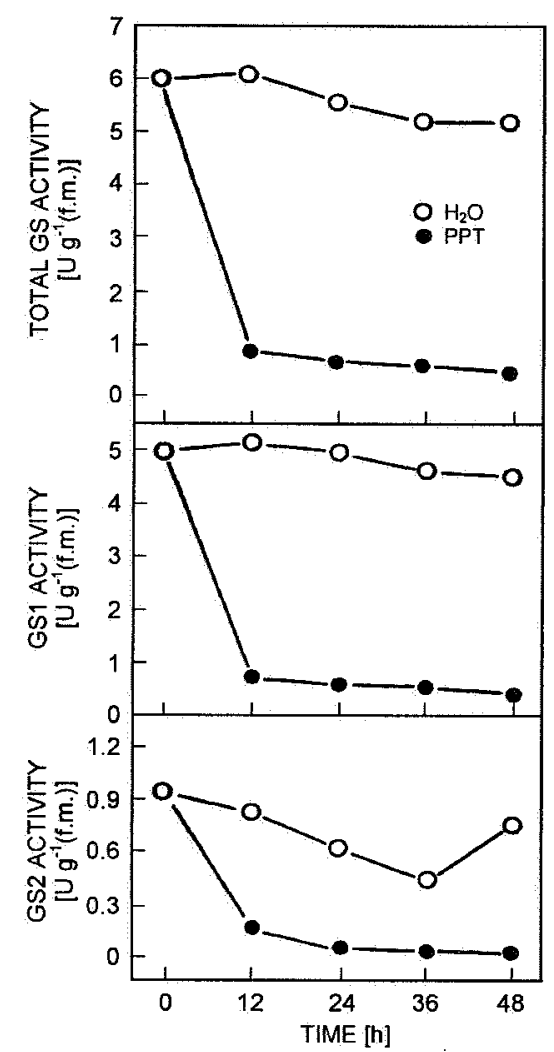

Fig. 5. Time courses of PPT effect on total GS, GS1, and GS2 activities in detached rice leaves in the light. Detached rice leaves were incubated in water or $50 \mu \mathrm{M}$ PPT. Vertical bars represent standard error $(n=4)$. Only those standard errors larger than the symbols are shown.

\section{References}

Bradford, M.M.: A rapid and sensitive method for the quantitation of microgram quantities of protein utilizing the principle of protein-dye binding. - Anal. Biochem. 72: 248254, 1976.

Givan, C.V.: Metabolic detoxification of ammonia in tissues of higher plants. - Phytochemistry 18: 375-382, 1979.

Hirel, B., Gadal, P.: Glutamine synthetase in rice. A comparative study of the enzyme from root and leaves. Plant Physiol. 66: 619-623, 1980.

Hurst, P.L., King, G.A., Borst, W.M.: Postharvest inhibition of glutamine synthetase activity with phosphinothricin reduces the shelf-life of asparagus. - Postharvest Biol. Technol. 3: 327-334, 1993.

Johnson, C.M., Stout, P.R., Broyer, T.C., Carlton, A.B.: reported that a steady decrease of GS2 was concomitant with the induction of GS1 in response to PPT treatment in the light.

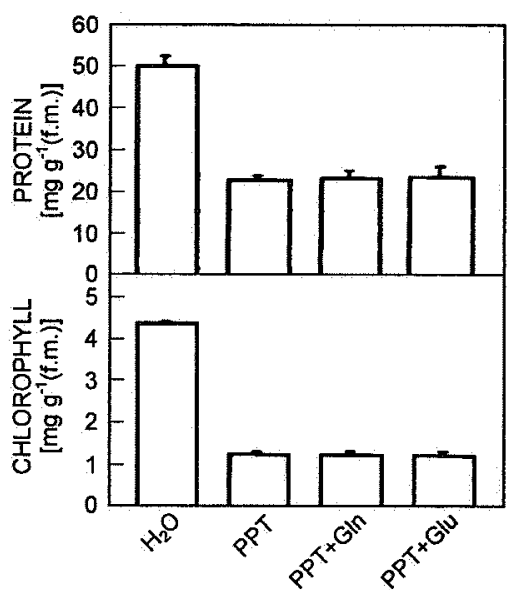

Fig. 6. Effects of glutamine (Gln) and glutamate (Glu) on PPTinduced toxicity in the light. Protein and chlorophyll contents were measured 3 days after treatment. Vertical bars represent standard errors $(n=4)$.

Inhibition of GS activity in PPT-treated detached rice leaves may also result in a decrease in glutamine (Gln) content. Lea et al. (1980) reported that PPT caused a sharp decrease in Gin content, when fed to leaves of Triticum, Pisum and Helianthus. Gln contents in rice leaves treated with PPT in the light for $3 \mathrm{~d}$ was found to be $10.6 \mu \mathrm{mol} \mathrm{g}^{-1}$ (f.m.), whereas Gln content in control leaves was $27 \mu \mathrm{mol} \mathrm{g}^{-1}$ (f.m.). Hurst et al. (1993) reported that Gln depletion rather than $\mathrm{NH}_{4}{ }^{+}$accumulation could be the reason for the reduced shelf-life of asparagus treated with PPT. In our work, addition of Gln or Glu (glutamate) had no effect on PPT-induced toxicity in the light (Fig. 6), it appears unlikely that lack of Gin is the reason for the toxicity of detached rice leaves treated with PPT in the light. Thus, the toxicity of PPT is a result of $\mathrm{NH}_{4}^{+}$accumulation in detached rice leaves.

Comparative choline requirements of different plant species. - Plant Soil 8: 337-353, 1957.

Lancien, M., Gadal, P., Hodges, M.: Enzyme redundancy and the importance of 2-oxoglutarate in higher plant ammoniun assimilation. - Plant Physiol. 123: 817-824, 2000.

Lea, P.J., Ridley, S.M.: Glutamine synthetase and its inhibition. - In: Dodge, A.D.(ed.): Herbicide and Plant Metabolism. Pp. 137-170. Cambridge University Press, New York 1990.

Lea, P.J., Joy, K.W., Ramos, J.P., Guerrero, M.G.; The action of 2-amino-4-(methylphosphinyl)-butanoic acid (phosphinothricin) and its 2-oxo-derivative on the metabolism of cyanobacteria and higher plants. - Phytochemistry 23: 1-6, 1980.

Lin, C.C., Kao, C.H.: Disturbed ammonium assimilation is 
associated with growth inhibition of roots in rice seedlings caused by NaCl. - Plant Growth Regul. 18: 233-238, 1996.

Lin, J.-N., Wang, J.-W., Kao, C.H.: Effect of abscisic acid and water stress on the senescence of detached rice leaves. Biol. Plant. 42: 313-316, 1999.

Lutts, S., Majerus, V., Kinet, J.-M.: NaCl effects on proline metabolism in rice (Oryza sativa) seedling. - Plant Physiol. 105: 450-458, 1999.

Lydon, J., Duke, S.O.: Inhibitors of glutamine biosynthesis, - In: Singh, B.K. (ed.): Plant Amino Acids. Pp. 445-464. Marcel Dekker, New York 1999.

Miflin, B.J., Lea, P.J.: The pathway of nitrogen assimilation in plants. - Phytochemistry 15: 873-885, 1976.

Oaks, A., Stulen, I., Jones, K., Winspear, M.J., Boesel, I.L.: Enzymes of nitrogen assimilation in maize roots. - Planta 148: $477-484,1980$.
Perez-Garcia, A., de Vicente, A., Canton, F.R., Cazorla, F.M., Codina, J.C., Garcia-Gutierrez, A., Canovas, F.M.: Lightdependent changes of tomato glutamine synthetase in response to Pseudomonas syringae infestion or phosphinothricin treatment. - Physiol. Plant. 102: 377-384, 1998.

Tachibana, K., Watanabe, T., Seikizawa, Y., Takematsu, T.: Accumulation of ammonia in plants treated with bialaphos. - J. Pestic. Sci. 11: 33-37, 1986.

Wild, A., Sauer, H., Ruhle, W.: The effect of phosphinothricin (glufosinate) on photosynthesis. I. Inhibition of photosynthesis and accumulation of ammonia. - $Z$. Naturforsch. 42c: 263-269, 1987.

Wintermans, J.F.G.M., De Mots, A.: Spectrophotometric characteristics of chlorophyll $a$ and $b$ and their pheophytins in ethanol. - Biochim. biophys. Acta 109: 448-453, 1965. 